\title{
MEASUREMENTS OF WAVE LENGTHS IN THE SPECTRA OF KRYPTON AND XENON
}

By Paul W. Merrill

\section{CONTENTS}

I. Introduction

II. Method of measurement.

III. Results.

r. Krypton.

2. Xenon.

IV. Discussion.

V. Summary

\section{INTRODUCTION}

The purpose of this paper is to record measurements of the wave lengths of lines in the red and infra-red spectral regions of krypton and xenon. The present measurements were made in continuation of considerable work upon the spectra of the rare gases already done by the Bureau of Standards. ${ }^{1}$

The rare gases are as follows:

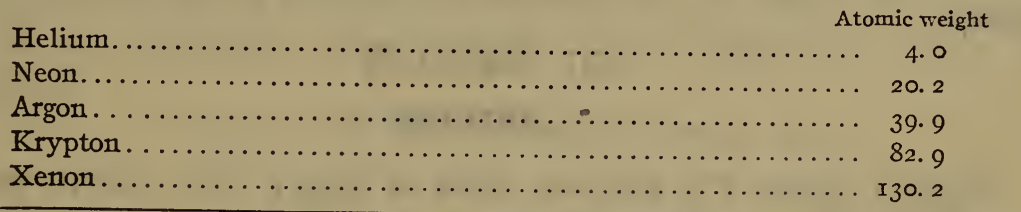

1 This Bulletin, 14, pp. 159 and 765; 1918, (Scientific Papers Nos. 302 and 329 ). $121191^{\circ}-19$ 
Published tables of wave lengths contain no xenon lines in the infra-red region and only two krypton lines. Thus these spectra have been practically unknown beyond the limits $6456 \mathrm{~A}$ for krypton and 6I98 A for xenon. Thanks to Baly, ${ }^{2}$ the spectra are quite well observed throughout shorter wave lengths. The other rare gases have been more thoroughly investigated. Since all of these elements are of particular interest to the spectroscopist it is desirable to complete our knowledge of them as rapidly as possible. Not only are their spectral lines suitable for working standards for wave length determinations of high accuracy, but they are important for theoretical reasons on account of the relative simplicity of their spectra, and because of certain very interesting numerical relations already discovered.

\section{METHODS OF MEASUREMENT}

The spectra were obtained with a concave grating giving a dispersion of ro angstroms per millimeter. The second order iron comparison spectrum gave the nearly normal dispersion curve from which the rare-gas wave lengths were interpolated. Owing to the long exposures and to the fact that there was no means of introducing the comparison spectrum during the exposure, several of the plates showed small systematic shifts. The resulting wave lengths, therefore, are not of a very high order of accuracy; the values based on three or more determinations should be within 0.02 or $0.03 \mathrm{~A}$ of the truth. In the tables which follow, those lines for which the uncertainty appears to be more than this are indicated by raising or omitting the second decimal figure.

\section{RESULTS}

\section{KRYPTON}

Table I shows the krypton lines of longer wave length measured in the first order. The two strongest lines in this region, $7587.40 \mathrm{~A}$ and $7601.55 \mathrm{~A}$, appear in Kayser's Handbuch as the two longest lines of the spectrum. In reality they are the beginning of a group of lines extending toward longer wave lengths, the krypton spectrum being analogous in this respect to that of argon and neon. 
TABLE 1.-Kr Lines Measured in First Order

\begin{tabular}{|c|c|c|c|c|c|c|c|}
\hline Intensity & I. A. & $\begin{array}{l}\text { Number } \\
\text { of ex- } \\
\text { posures }\end{array}$ & Baly & Intensity & I. A. & $\begin{array}{c}\text { Number } \\
\text { of ex- } \\
\text { posures }\end{array}$ & Runge \\
\hline 3 & 6421.1 & 1 & 6421.11 & 0 & 7635.1 & 1 & \\
\hline 5 & $6456.3^{4}$ & 1 & 6456.44 & 7 & 7685.22 & 5 & \\
\hline 1 & $6576.4^{2}$ & 1 & .......... & 8 & 7694.53 & 5 & \\
\hline 2 & $6652.2^{\circ}$ & 1 & ............ & 0 & 7741.3 & 1 & \\
\hline 3 & $6699.1^{4}$ & 1 & ........... & 2 & 7746.81 & 3 & \\
\hline 0 & 6739.9 & 1 & ........... & 0 & 7776.2 & 1 & \\
\hline 3 & $6813.0^{8}$ & 1 & ........... & 1 & $7806.5^{8}$ & 1 & \\
\hline 0 & 6846.4 & 1 & .... & 7 & 7854.79 & 5 & \\
\hline 1 & $6869.6^{0}$ & 1 & & 2 & 7913. 38 & 3 & \\
\hline 5 & $6904.6^{4}$ & 1 & & 2 & $7928.5^{\circ}$ & 3 & \\
\hline 2 & 7224.00 & 3 & ........... & 0 & 7982.4 & 1 & \\
\hline 2 & 7287.27 & 4 & & 4 & 8059.47 & 5 & \\
\hline 2 & 7425.52 & 4 & ....... & 7 & 8104.33 & 5 & \\
\hline 3 & 7486.85 & 5 & ...... & 10 & 8112.87 & 5 & \\
\hline 1 & 7493.6 & 1 & ........ & 6 & 8190.02 & 5 & \\
\hline 1 & 7494.1 & 1 & & 4 & 8263.22 & 5 & \\
\hline 0 & 7503.9 & 1 & n.......... & 3 & 8281.02 & 5 & \\
\hline 0 & 7514.7 & 1 & Runge & 6 & 8298.07 & 5 & \\
\hline 15 & 7587.40 & 6 & 7587.20 & 2 & 8508.85 & 5 & \\
\hline \multirow[t]{2}{*}{20} & 7601.55 & 6 & 7601.19 & 3 & 8776.73 & 5 & \\
\hline & & & & 1 & 8928.72 & 5 & \\
\hline
\end{tabular}

The krypton tubes, which were furnished by Sir William Ramsey, contained krypton of a high degree of purity. Two argon lines are doubtfully present in the spectrum, while the strongest line of the xenon spectrum, $8231.62 \mathrm{~A}$, shows faintly.

For the purpose of comparing the gas in these tubes with that observed by Baly, one or two short exposures were made in the blue, using the second order spectrum. The few lines obtained correspond with the strongest lines observed by Baly ${ }^{3}$ and Runge, ${ }^{4}$ while no extraneous lines appear. The results are given in Table 2.

TABLE 2. $-\mathrm{Kr}$ Lines Measured in Second Order

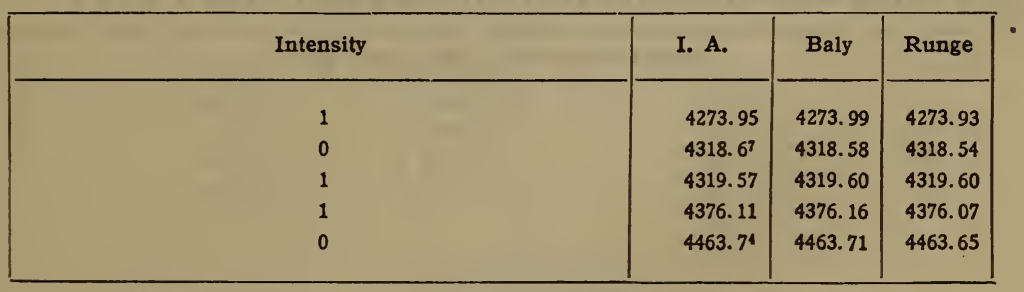

${ }^{3}$ Baly, loc. cit.

4 Runge, Astroph. J1., 10, p. 73; 1899. 
A very brief inspection of the wave lengths for numerical regularities has shown three new pairs of constant frequency differences of the type discovered by Paulson. ${ }^{5}$ The data for all eight of these pairs are given in Table 3 .

TABLE 3.-Frequency Differences in Krypton Spectrum

\begin{tabular}{|c|c||c|c|}
\hline I. A. & $\Delta \mathbf{n}$ vacuum & I. A. & $\Delta \mathbf{n}$ vacuum \\
\hline 4273.99 & 945.04 & 5570.28 & 944.99 \\
4453.95 & & 5879.84 & \\
4283.01 & 944.95 & 7601.55 & 944.97 \\
4463.71 & & 8190.02 & \\
4318.58 & 945.06 & 7694.53 & 945.00 \\
4502.39 & & 8298.07 & \\
5562.23 & 944.96 & 8104.33 & 945.06 \\
5870.90 & & 8776.73 & \\
\hline
\end{tabular}

The mean value of the frequency difference is 945.00. The greatest divergence from this value is 0.06 , which is one part in 200000 of the frequency. This is probably not outside the errors of measurement, especially if we assume the error of a difference to be 1.4 times that of an individual line. It would be of great interest to measure these lines with a much higher accuracy to see whether the differences remain constant to a part in several million, as has been found to be the case with neon.

\section{XENON}

The xenon as supplied by the laboratories of Sir William Ramsey was very pure. In the regions shown by the tables to overlap with Baly's measurements, the spectrum corresponds to that observed by him. Some plates taken by F. M. Walters, jr., with a I-prism spectrograph covering a long range of the shorter wave lengths, also show a reasonable correspondence with Baly's spectra. The grating measurements are contained in Tables 4 and 5 . 
TABLE 4.-Xe Lines Measured in First Order

\begin{tabular}{|c|c|c|c|c|c|c|c|}
\hline Intensity & I. A. & $\begin{array}{l}\text { Number } \\
\text { of ex- } \\
\text { posures }\end{array}$ & Baly & Intensity & I. A. & $\begin{array}{c}\text { Number } \\
\text { of ex- } \\
\text { posures }\end{array}$ & Baly \\
\hline 1 & $5823.9^{8}$ & 1 & 5823.86 & 0 & 7266.4 & 1 & \\
\hline 0 & 5824.9 & 1 & 5824.76 & 1 & 7283.90 & 2 & \\
\hline 1 & $5875.0^{2}$ & 1 & 5875.08 & 3 & $7285.3^{6}$ & 2 & \\
\hline 1 & 5895.01 & 1 & 5894.98 & 2 & $7316.3^{2}$ & 2 & \\
\hline 0 & 5931.2 & 1 & .......... & 2 & $7321.4^{8}$ & 2 & \\
\hline 1 & $5934.2^{2}$ & 1 & $\ldots \ldots \ldots$ & 2 & $7336.4^{4}$ & 2 & \\
\hline 0 & 6163.8 & 1 & 6164.09 & 1 & $7355.6^{2}$ & 2 & \\
\hline 1 & $6178.3^{2}$ & 1 & 6178.59 & 2 & $7385.9^{7}$ & 2 & \\
\hline 0 & 6179.6 & 1 & 6179.95 & 3 & $7393.8^{\circ}$ & 2 & \\
\hline 2 & $6182.4^{4}$ & 1 & 6182.71 & 1 & $7400.4^{1}$ & 2 & \\
\hline 1 & $6198.3^{0}$ & 1 & 6198.49 & 0 & $7424.0^{2}$ & 2 & \\
\hline 3 & $6318.0^{6}$ & 1 & & 1 & $7472.0^{6}$ & 2 & \\
\hline 3 & $6469.7^{\circ}$ & 1 & & 0 & $7473.9^{9}$ & 2 & \\
\hline 1 & $6472.8^{2}$ & 1 & & 0 & 7559.7 & 2 & \\
\hline 1 & $6487.7^{4}$ & 1 & & 2 & $7584.6^{8}$ & 4 & \\
\hline 0 & 6498.7 & 1 & & 4 & 7642.04 & 5 & \\
\hline 0 & 6504.1 & 1 & & 1 & $7643.8^{5}$ & 4 & \\
\hline 1 & $6595.5^{4}$ & 1 & & 0 & 7664.3 & 1 & \\
\hline 0 & 6666.8 & 2 & & 1 & $7802.7^{0}$ & 3 & \\
\hline 2 & $6668.8^{0}$ & 2 & & 3 & $7887.4^{2}$ & 4 & \\
\hline 3 & 6727.90 & 2 & & 2 & 7967.34 & 4 & \\
\hline 1 & $6827.1^{9}$ & 1 & & 1 & $8057.2^{7}$ & 3 & \\
\hline 0 & 6866.6 & 1 & & 1 & $8061.3^{4}$ & 3 & \\
\hline 1 & $6872.0^{6}$ & 1 & & 2 & 8206.30 & 4 & \\
\hline 3 & $6882.0^{7}$ & 2 & & 15 & 8231.62 & 6 & \\
\hline 0 & 6976.1 & 1 & ...... & 1 & $8266.5^{2}$ & 3 & \\
\hline 0 & 7047.2 & 1 & 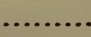 & 12 & 8280.08 & 6 & \\
\hline 2 & $7119.5^{8}$ & 3 & & 3 & 8346.76 & 4 & \\
\hline 1 & $7257.9^{4}$ & 1 & & 4 & 8409.17 & 4 & \\
\hline 0 & 7262.6 & 1 & $\ldots$ & 6 & 8819. 38 & 4 & \\
\hline $1=$ & & & & 1 & 8952.25 & 4 & \\
\hline & & & & 1 & 9045.44 & 3 & \\
\hline & & & & 0 & $9162.7^{6}$ & 2 & \\
\hline
\end{tabular}

TABLE 5.-Xe Lines Measured in Second Order

\begin{tabular}{|c|c|c|c||c|c|c|c|}
\hline Intensity & I. A. & $\begin{array}{c}\text { Number } \\
\text { of ex- } \\
\text { posures }\end{array}$ & Baly & Intensity & I. A. & $\begin{array}{c}\text { Number } \\
\text { of ex- } \\
\text { posures }\end{array}$ & Baly \\
\hline \multirow{2}{*}{} & $a(3967.6)$ & 1 & 3967.59 & 10 & 4671.227 & 3 & 4671.24 \\
3 & 4500.98 & 2 & 4500.96 & 3 & 4671.56 & 2 & $\ldots \ldots \ldots .$. \\
1 & 4524.70 & 2 & 4524.65 & 1 & 4697.02 & 2 & 4696.99 \\
6 & 4624.276 & 3 & 4624.28 & 2 & 4734.18 & 2 & 4734.12 \\
0 & 4624.58 & 2 & $\ldots \ldots \ldots .$. & 1 & 4807.06 & 2 & 4807.01 \\
& & & & & 4807.65 & 1 & $\ldots \ldots \ldots .$. \\
\hline
\end{tabular}

a Position estimated. 
By far the strongest lines in the region 5823-9162 are 8231.62 and 8280.08. These two lines seem to correspond to the strong lines which stand at the short wave length end of groups of lines of the other rare gases. They are certainly among the most important lines in the whole spectrum, possibly being those by which traces of the elements could be most easily identified. The line 823I was measured three times on the krypton photographs, yielding a value of 8231.62 , in exact agreement with the wave length from the xenon tube.

Attention is called to the close companions to the lines 4624 , $467 \mathrm{I}$, and 4807 . These may have been lost in the over-exposed images of the strong lines on Baly's plates.

\section{DISCUSSION}

Although all the principal lines of the helium spectrum have been shown to fall unmistakably into six series, no important series relationships have as yet been found among the stronger lines of the other rare gases. While it would be rash to say they do not exist, a different type of regularity is predominant in present data-namely, that of constant difference pairs. The four gases of greater atomic weight possess another feature in common which is apparently not partaken of by the helium spectrum. That is the tendency of the lines to form groups, the position of which apparently has some relation to the atomic weight. Observations upon neon and argon had previously showil that each spectrum possessed a group of very strong lines in the red, with the further characteristic that the shortest lines of the group were especially strong, and that in the direction of the violet from these lines lay a region containing only weak lines. As has already been remarked in this paper, the krypton spectrum exhibits somewhat the same phenomena. This is probably also the case with xenon, the very strong lines 823I and 8280 beginning a group of strong lines, which, however, is not very conspicuous or greatly extended owing to the rapidly decreasing efficiency of the photographic plate in this portion of the spectrum.

Moreover, strong lines or groups of the different gases show a regular displacement from one spectrum to another in the order of the atomic weights. To exhibit this the writer photographed the four rare gases side by side upon the same plate with a very small dispersion spectrograph. The similarity of the spectra and the progression in the position of two groups of lines is shown by Fig. I, 
Scientific Papers of the Bureau of Standards, Vol. 15

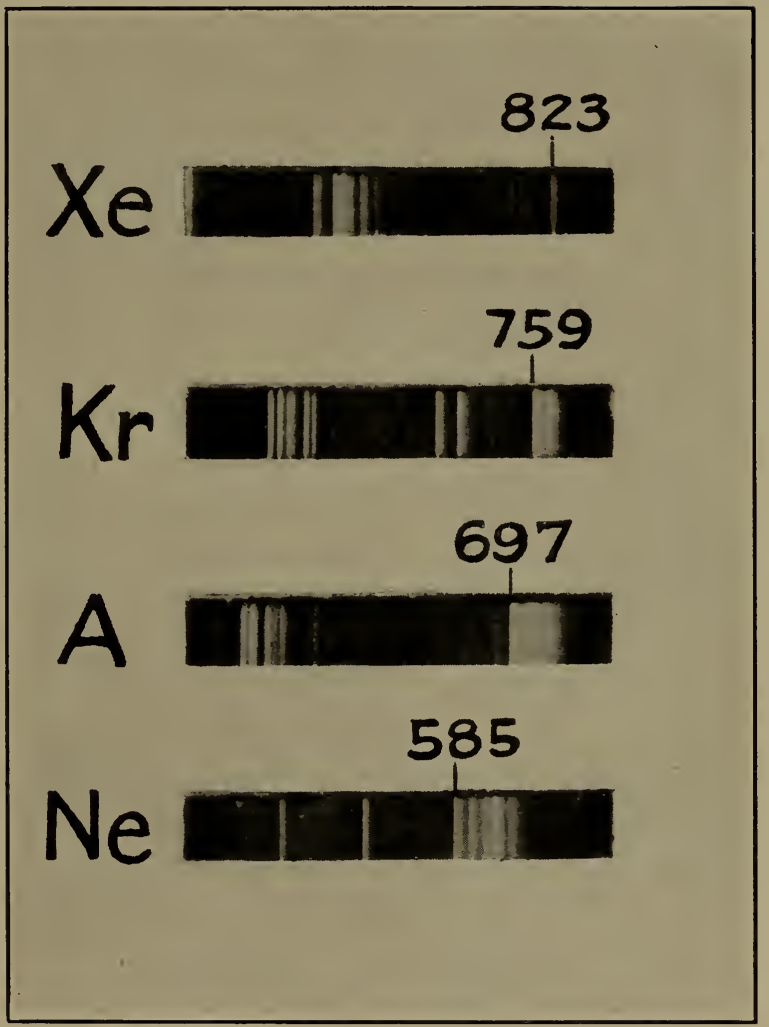

FIG. I.-Spectra of the four rare gases showing similarity and progression in the group position 
which is a copy of this photograph. Bearing in mind that the long wave-length group in xenon is much weakened by photographic effects, and that the short wave-length group of neon fails to appear on account of the absorption of the prism used, the correspondence is rather striking. The correspondence between individual lines is not clear. No extended search for individual relationships has as yet been made. A few simple functions of atomic weight and number have been tried to represent the position of the lines. The writer is not convinced that the apparent success in a few cases indicates anything of importance, as it may be due to a few coincidences. At any event no complete system has been evolved and it remains to be seen whether such relationships exist.

F. M. Walters made the photographs to check the character of the krypton and xenon spectra in the violet and ultra-violet and assisted in some of the wave-length reductions.

\section{SUMMARY}

This paper records photographic measurements of wave lengths in the spectra of krypton and xenon, principally in the red and infra-red. In krypton 37 new lines were measured between $6576 \mathrm{~A}$ and $8928 \mathrm{~A}$; in xenon 52 new lines between $6318 \mathrm{~A}$ and $9162 \mathrm{~A}$. In this region there are numerous strong lines which are probably among the most important in the spectra of these elements. The xenon lines at 823I A and 8280 A are especially notable. These and other lines may be of value as wave-length standards in the infra-red.

Attention is called to a probable analogy between the spectra of the rare gases neon, argon, krypton, and xenon which this investigation has brought to light.

Washington, April 28, I9I9. 


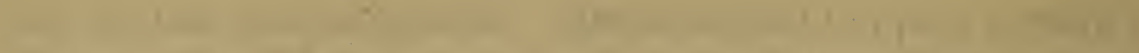

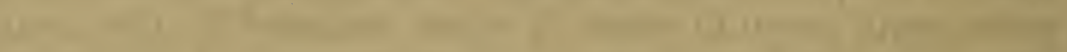

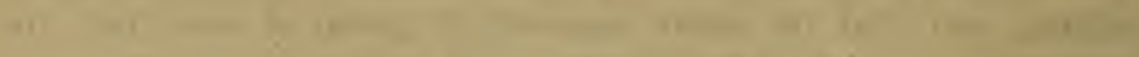

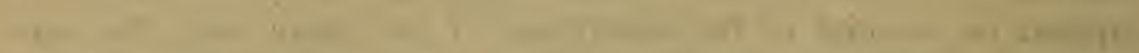

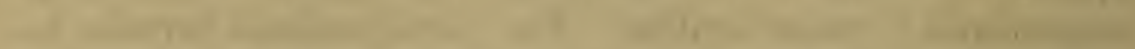

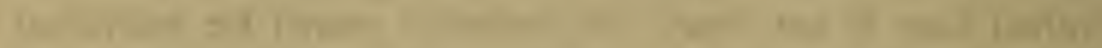

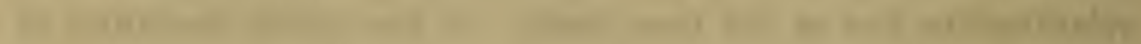

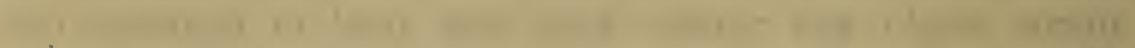

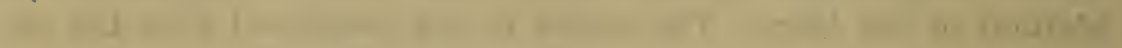

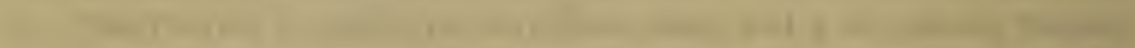

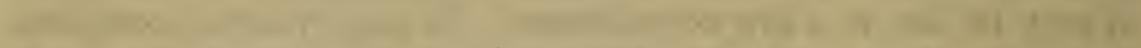
H.

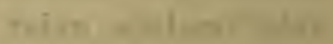

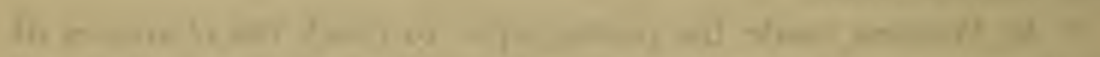

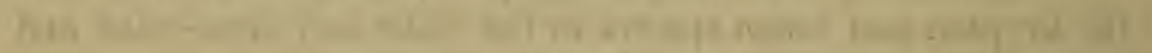

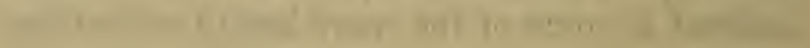

\section{anititing}

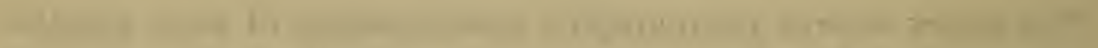

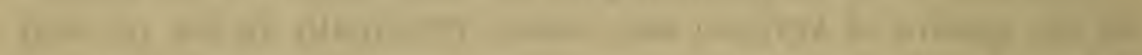

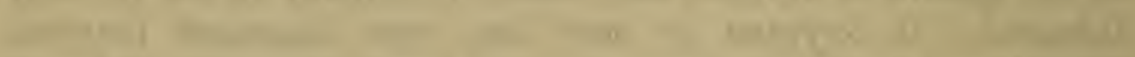

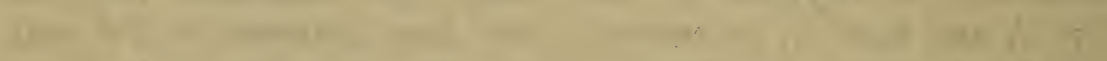

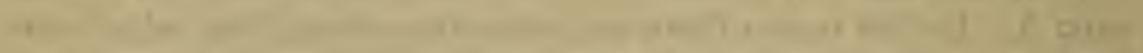

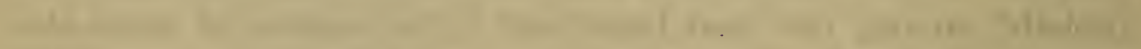

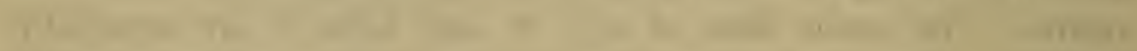

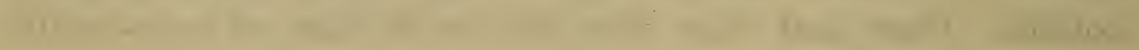

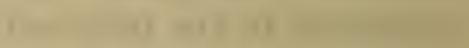

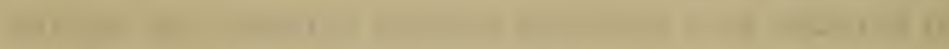

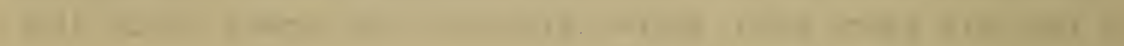

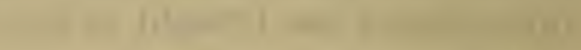

$$
\text { ant at is }
$$




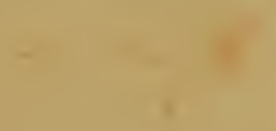


